\title{
Oxytocin Modulates Social Distance between Males and Females
}

\author{
Dirk Scheele, ${ }^{1 \star}$ Nadine Striepens, ${ }^{1 \star}$ Onur Güntürkün, ${ }^{2}$ Sandra Deutschländer, ${ }^{1}$ Wolfgang Maier, ${ }^{1,4}$ Keith M. Kendrick, ${ }^{{ }^{\dagger}}$ \\ and René Hurlemann ${ }^{1 \dagger}$ \\ ${ }^{1}$ Department of Psychiatry, University of Bonn, 53105 Bonn, Germany, ${ }^{2}$ Department of Biopsychology, Ruhr University, 44780 Bochum, Germany, ${ }^{3}$ Key \\ Laboratory for Neuroinformation, School of Life Science and Technology, University of Electronic Science and Technology of China, 610054 Chengdu, P.R. \\ China, and ${ }^{4}$ German Center for Neurodegenerative Diseases, 53175 Bonn, Germany
}

In humans, interpersonal romantic attraction and the subsequent development of monogamous pair-bonds is substantially predicted by influential impressions formed during first encounters. The prosocial neuropeptide oxytocin (OXT) has been identified as a key facilitator of both interpersonal attraction and the formation of parental attachment. However, whether OXT contributes to the maintenance of monogamous bonds after they have been formed is unclear. In this randomized placebo-controlled trial, we provide the first behavioral evidence that the intranasal administration of OXT stimulates men in a monogamous relationship, but not single ones, to keep a much greater distance $(\sim 10-15 \mathrm{~cm})$ between themselves and an attractive woman during a first encounter. This avoidance of close personal proximity occurred in the physical presence of female but not male experimenters and was independent of gaze direction and whether the female experimenter or the subject was moving. We further confirmed this unexpected finding using a photograph-based approach/ avoidance task that showed again that OXT only stimulated men in a monogamous relationship to approach pictures of attractive women more slowly. Importantly, these changes cannot be attributed to OXT altering the attitude of monogamous men toward attractive women or their judgments of and arousal by pictures of them. Together, our results suggest that where OXT release is stimulated during a monogamous relationship, it may additionally promote its maintenance by making men avoid signaling romantic interest to other women through close-approach behavior during social encounters. In this way, OXT may help to promote fidelity within monogamous human relationships.

\section{Introduction}

Humans and other mammalian species exhibiting monogamous pair-bonds also show biparental behavior, leading to the theory that while reproductively costly for males, monogamy evolves in species to help ensure offspring survival (Low, 2003). Research on monogamous mammals, particularly prairie voles, has shown that the neuropeptide oxytocin (OXT) promotes the formation of pair-bonds in females, with a related peptide, argininevasopressin being more important in males (Donaldson and Young, 2008; Insel, 2010). In humans, OXT has also been implicated in attraction and bonding behavior in both men and women (Meyer-Lindenberg et al., 2011; Feldman, 2012; McCall and Singer, 2012). While it has been reported to reduce conflict

\footnotetext{
Received June 8, 2012; revised Sept. 6, 2012; accepted Sept. 11, 2012.

Author contributions: D.S., N.S., K.M.K., and R.H. designed research;D.S., N.S., and S.D. performed research; D.S., N.S., S.D., and R.H. analyzed data; D.S., N.S., O.G., W.M., K.M.K., and R.H. wrote the paper.

This work was supported by a German Research Foundation grant (HU1302/2-2) and by a Starting Independent Researcher Grant (NEMO — Neuromodulation of Emotion) jointly provided by the Ministry of Innovation, Science, Research and Technology of the German State of North Rhine-Westphalia and the University of Bonn (to R.H.). We thank Paul Jung and Hani Quadai for superb research assistance.

The authors declare no competing financial interests.

${ }^{*}$ D.S. and N.S. contributed equally to this work.

${ }^{\dagger}$ K.M.K. and R.H. share senior authorship.

Correspondence should be addressed to Dr. René Hurlemann, Department of Psychiatry, University of Bonn, 53105 Bonn, Germany. E-mail: renehurlemann@me.com.

DOI:10.1523/JNEUROSCI.2755-12.2012

Copyright $\odot 2012$ the authors $\quad 0270-6474 / 12 / 3216074-06 \$ 15.00 / 0$
}

between couples (Ditzen et al., 2009), this may be a generalized effect of its prosocial actions independent of relationship status. To date, there has been no direct evidence that OXT release after the formation of monogamous bonds actually contributes to their subsequent maintenance.

We signal interest in members of the opposite sex in many ways during influential first encounters (Sunnafrank and Ramirez, 2004), although approach behavior is clearly important for displaying potential romantic interest. The personal space (PS) we keep between us and others is crucially influenced by our relationships with them; a closer, intimate zone being reserved for lovers. However, if an unfamiliar person violates our PS (Hall, 1966; Hayduk, 1983), we can feel uncomfortable, and so close approach must be carefully controlled. The amygdala may be important for this regulation (Kennedy et al., 2009) and the activity of amygdala subregions is also modulated by OXT (Gamer et al., 2010). Furthermore, OXT is known to increase gaze directed at the eyes of others (Guastella et al., 2008) and we tend to expand our PS if a person establishes eye-contact with us (Argyle and Dean, 1965). The prosocial actions of OXT often involve facilitation of approach behaviors (Hurlemann et al., 2010; Kemp and Guastella, 2011; Striepens et al., 2011), although this can be context- and person-dependent (Bartz et al., 2011), since OXT can also promote risk aversion (Declerck et al., 2010) and noncooperation toward individuals perceived as outsiders (De Dreu, 2012). 
Given this empirical background, we hypothesized that in addition to individual trait characteristics such as social cognitive proficiency and attachment anxiety, relationship status may be a critical factor in determining the influence of OXT on the willingness of men to approach unfamiliar women, evident either in a reduced or enlarged PS. To determine the direction of this hypothesized effect, we measured the distance single and pairbonded men keep from an unfamiliar attractive woman who either maintains or avoids eye contact with them during a first encounter, and also assessed their willingness to approach or avoid pictures of attractive women. In addition, we conducted an independent control experiment involving a male experimenter and pair-bonded men to disambiguate a general effect of OXT on social interactions from a specific effect on pairbond maintenance.

\section{Materials and Methods}

Subjects and protocols. A total of 86 healthy heterosexual male adults participated in this study after giving written, informed consent. For Experiments 1 and 2, 57 subjects were enrolled in one cohort. A second cohort including 29 subjects was tested in an independent control experiment. The study was approved by the institutional review board of the Medical Faculty of the University of Bonn and was performed in compliance with the latest revision of the Declaration of Helsinki. Subjects were free of current and past physical or psychiatric illness, as assessed by medical history and a Structured Clinical Interview for DSM-IV axis I (SCID-I) and axis II disorders (SCID-II). In addition, they were naive to prescription-strength psychoactive medication and had not taken any over-the-counter psychoactive medication in the past 4 weeks. The participants were asked to maintain their regular sleep and waking times and to abstain from caffeine and alcohol intake on the day of the experiment.

All subjects completed a comprehensive neuropsychological test battery to control for possible pretreatment differences in cognitive performance. We also assessed personality traits that might affect the attitude toward social distance by using, for instance, the Social Interaction Anxiety Scale and the Social Phobia Scale (Mattick and Clarke, 1998). Additionally, we measured mood and state anxiety before and after the experimental tasks.

Using a double-blind, randomized, parallel group design, we administered either intranasal OXT (24 IU, Syntocinon-Spray, Novartis; 3 puffs per nostril, each with 4 IU OXT) or placebo (PLC; sodium chloride solution) to 57 healthy heterosexual male volunteers $($ mean $\pm \mathrm{SD}$; age $=$ $25.1 \pm 3.3$ years) who were either single $(n=27)$ or in a stable monogamous relationship ( $n=30), 45 \mathrm{~min}$ before the start of the experiments. The ratio of singles and subjects in a relationship was comparable between the OXT and PLC groups $\left(\chi_{(1)}^{2}=0.15, p=0.70\right)$. In the first experiment, we used the stop-distance paradigm to determine both the ideal distance ( = PS) for an interaction with an attractive female experimenter (S.D.) and the distance at which the subjects felt slightly uncomfortable (Fig. 1 Ai). Next, the subjects were asked to choose the distance that an average person would regard as optimal and to rate on a 1-9 scale the sympathy and trustworthiness of the experimenter as well as their feelings during the testing (valence, arousal, embarrassment, and attention toward their own emotions). In the second experiment, subjects performed an approach/avoidance task (AA-task) measuring the speed and errors of manual approach and avoidance responses to stimuli varying both on valence and social content. Subjects had to discriminate between positive (attractive women or beautiful landscapes) and negative (mutilations or dirt) pictures selected from the International Affective Picture System (IAPS) (Lang et al., 2005) as fast as possible by pulling a joystick toward (positive) or away (negative) from their own body. After the AA-task, all subjects were administered the Self-Assessment Manikin (Lang et al., 2005) to obtain self-reported pleasure and arousal ratings for each stimulus on a scale ranging from 1 (minimum) to 9 (maximum). In addition, we used the stop-distance paradigm in a double-blind randomized control experiment involving a male experimenter (H.Q.) and another 29 healthy heterosexual pair-bonded men
$(25.9 \pm 3.2$ years $)$ who were administered either OXT $(n=14)$ or PLC $(n=15)$. Since Experiments 1 and 2 included no attractiveness ratings of the female experimenter (S.D.), a photograph of her was presented to the participants of the independent control experiment and they were asked to judge her attractiveness on a visual analog scale ranging from 0 (minimum) to 100 (maximum).

Stop-distance paradigm. We applied an adapted version of the stopdistance paradigm used by Kennedy et al. (2009). All subjects read written instructions before the experiment and met the experimenter for the first time. Care was taken to assure the same appearance of the experimenter across all sessions, and all subjects were tested in the same room. All trials were administered in a fixed order, and all subjects completed a practice trial before the start of the experiment. Subjects were positioned at one end of the room with their toes on a line that was marked on the floor. In the first half of the trials, the female experimenter was the one moving at a natural gait either toward ("far", i.e., start distance of $2 \mathrm{~m}$ ) or away ("close", start distance of $30 \mathrm{~cm}$ ) from the subject, whereas in the second half, the male volunteer was the one moving. This experimental variation served to mimic approach or withdrawal during influential first encounters. The experimenter avoided eye-contact in half the trials, although subjects were not informed of this during initial task instruction. Subjects were asked to tell the experimenter to stop at their preferred distance in the first half of the trials and choose their ideal distance in the second half of the trials when they were moving. This was fine-tuned as subjects could have the experimenter move slightly further backward or forward. After determining the ideal distance in trials with a far start distance, the experimenter or the subject came even closer until the subject felt slightly uncomfortable. The final chin-to-chin distance was measured with a digital laser measurer (model DLR165K; Bosch; error $\pm 0.003 \mathrm{~m})$.

Approach/avoidance task. The pictures of the task were adjusted to closely resemble each other in luminance using a self-written script in Matlab 7 (MathWorks). Each trial started with the presentation of a fixation cross for between 1 and $2 \mathrm{~s}$ and then the pictures were presented for $2000 \mathrm{~ms}$. The pictures were administered in four blocks, each containing five positive social, positive nonsocial, negative social, and negative nonsocial pictures in a random order. The participants were asked to place their head on a chin rest at a viewing distance of $\sim 50 \mathrm{~cm}$ to the computer screen. Approach and avoidance behavior were simulated by increasing or decreasing the picture size. Pulling the joystick replaced the picture by the same one enlarged by a factor of 1.1, while pushing the joystick reduced the picture size by a factor of 0.9 . Errors occurring in the form of joystick moves in the wrong direction were recorded and analyzed separately. The reaction time was obtained by using the joystick displacement measurements for each trial. Trials showing an extreme reaction time $(>1500 \mathrm{~ms})$ or movements in the wrong direction were excluded. All subjects completed six practice trials before the start of the experiment.

\section{Results}

Groups did not differ in any of the demographic, neuropsychological, or personality characteristics listed in Tables 1 and 2. For Experiment 1, a repeated-measures ANOVA with "person moving" (experimenter or subject), "starting position" (far or close), and "eye contact" (yes or no) as within-subjects factors, "treatment" (OXT or PLC) and "relationship status" (pair-bonded or single) as between-subjects factors, and "PS" as a dependent variable revealed a main effect (across all conditions) of treatment $\left(F_{(1,53)}=10.62, p<0.01, \eta^{2}=0.17\right)$ and relationship status $\left(F_{(1,53)}=5.41, p=0.02, \eta^{2}=0.09\right)$ and an interaction between treatment and relationship status $\left(F_{(1,53)}=4.73, p=0.03, \eta^{2}=\right.$ 0.08 ; Fig. 1 Aii,Aiii). Post hoc unpaired $t$ tests showed that PS was significantly increased in pair-bonded men $\left(t_{(28)}=-4.07, p<\right.$ $\left.0.01, \eta^{2}=0.37\right)$ but not in single men $\left(t_{(25)}=-0.72, p=0.48\right.$, $\left.\eta^{2}=0.02\right)$. These results cannot be interpreted in terms of altered distance perception since there was no OXT effect $(p=$ 
$A_{(i)}$
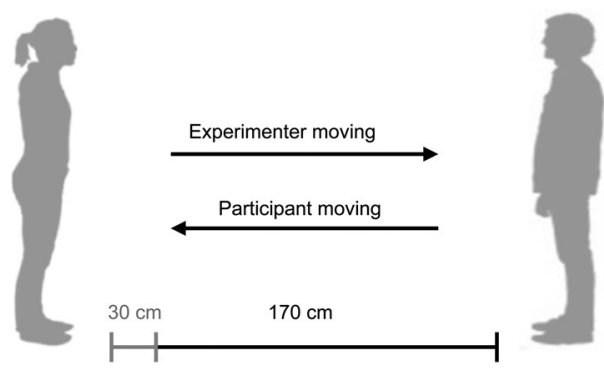

(iii)

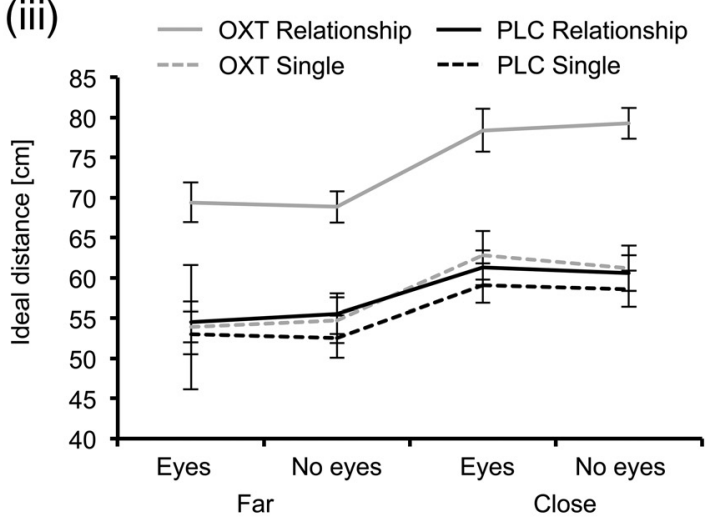

B

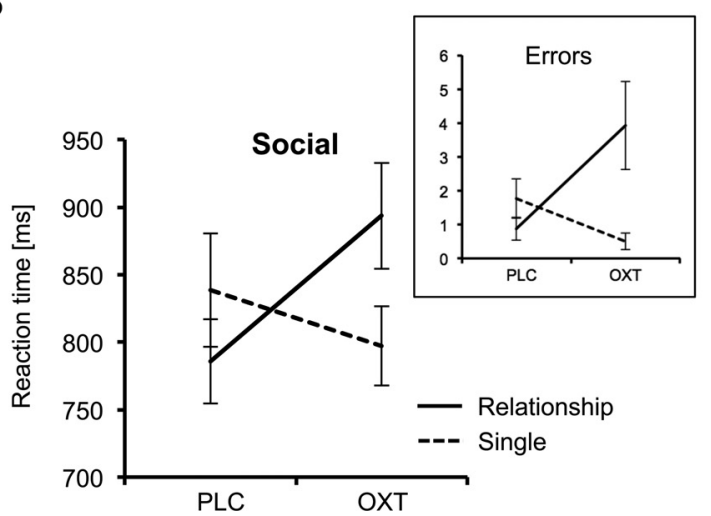

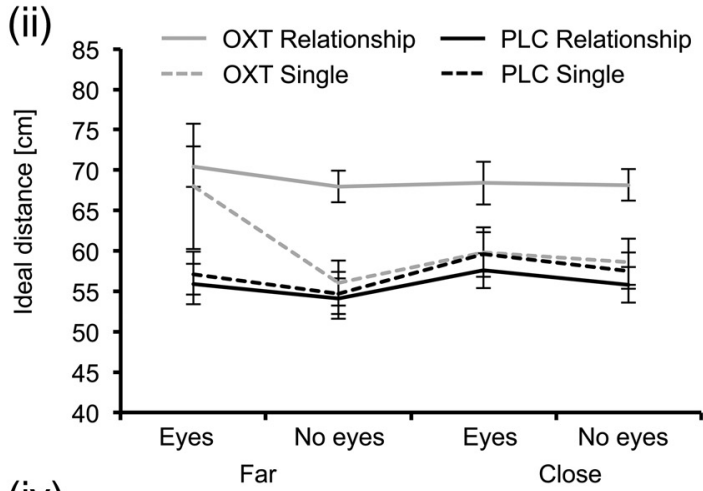

(iv)
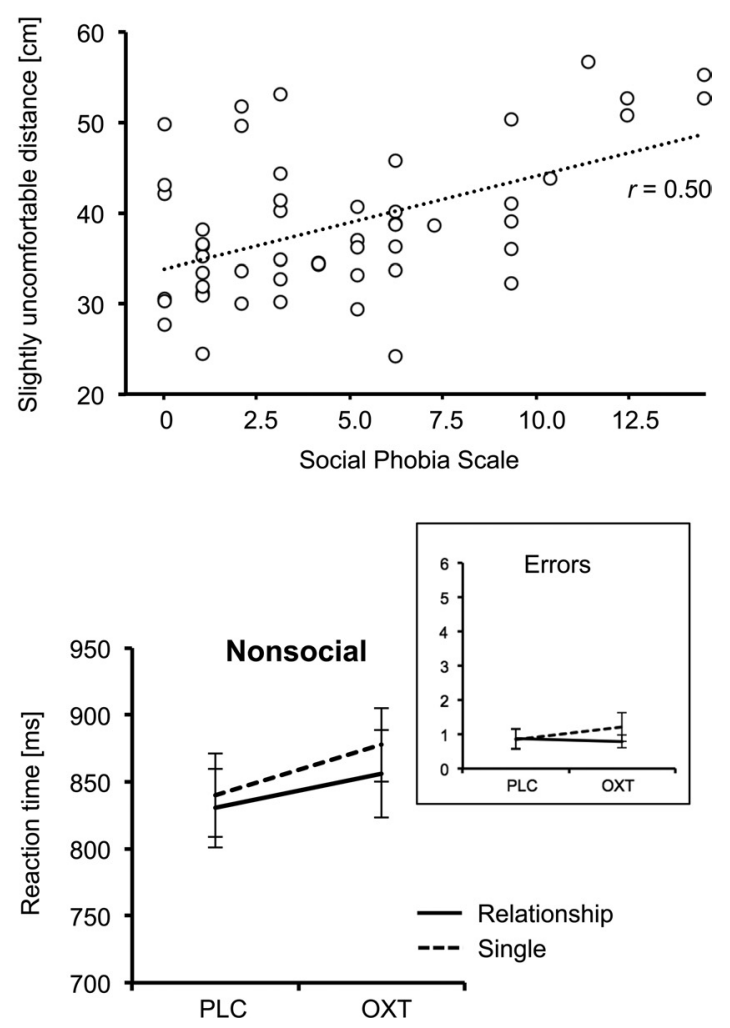

Figure 1. Effects of OXT in the stop-distance paradigm $(\boldsymbol{A})$ and in the approach-avoidance task $(\boldsymbol{B})$. $\boldsymbol{A} \boldsymbol{i}$, Experimental setup. In the first half of the trials, the female experimenter was the one moving either toward ("far", i.e., start distance of $2 \mathrm{~m}$ ) or away ("close", start distance of $30 \mathrm{~cm}$ ) from the subject; in the second half, the male volunteer was the one approaching or withdrawing. An additional condition was gaze direction, with the female experimenter avoiding eye contact in half of the trials. Aii, Ideal distances when the experimenter was moving. Aiii, Ideal distances when the subject was moving. Across all conditions, OXT increased the ideal distance that pair-bonded men maintained in relation to the unknown attractive woman. Aiv, Slightly uncomfortable distances are positively correlated with social phobia scores across groups. For the approach-avoidance task, response times and number of error trials are shown. $\boldsymbol{B}$, Reaction times (ms) of correct trials and number of error trials for the approach of positive, social and nonsocial pictures. In the OXT group, subjects in a relationship pulled the stimuli significantly slower toward themselves and made significantly more errors than singles only with social pictures. Error bars indicate the SEM.

0.41) on the subjects' estimation of the ideal distance for an average person.

Furthermore, the repeated-measures ANOVA yielded a main effect of starting position $\left(F_{(1,53)}=27.20, p<0.01, \eta^{2}=0.34\right)$ as well as an interaction of starting position and person moving $\left(F_{(1,53)}=32.33, p<0.01, \eta^{2}=0.38\right)$. Indeed, PS was larger when subjects were moving away $(65.12 \pm 14.62 \mathrm{~cm})$ from rather than toward $(57.81 \pm 13.51 \mathrm{~cm})$ the experimenter, suggesting that a proximal starting position was felt as more unpleasant than a distal one. There was also a small but significant main effect of eye contact $\left(F_{(1,53)}=7.81, p<0.01, \eta^{2}=0.13\right)$ showing larger distances in trials with eye contact $(61.75 \pm 11.86 \mathrm{~cm})$ than in trials with averted gaze $(60.22 \pm 11.48 \mathrm{~cm})$. A significant inter- action of eye contact and person moving $\left(F_{(1,53)}=7.05, p=0.01\right.$, $\left.\eta^{2}=0.11\right)$ revealed that this effect was stronger if the experimenter $(61.97 \pm 12.06 \mathrm{~cm}$ vs $59.02 \pm 10.24 \mathrm{~cm})$ instead of the subject $(61.52 \pm 14.07 \mathrm{~cm}$ vs $61.41 \pm 13.83 \mathrm{~cm})$ was moving. Finally, there was an effect of relationship status, with pairbonded men keeping more distance than singles and which also depended on the person moving $\left(F_{(1,53)}=6.17, p=0.02, \eta^{2}=\right.$ $0.10)$. The difference between singles and pair-bonded men was stronger in trials where the subject was moving $(57.03 \pm 12.12$ $\mathrm{cm}$ vs $65.45 \pm 14.35 \mathrm{~cm}$ ) compared with trials where the experimenter was moving $(58.98 \pm 10.29 \mathrm{~cm}$ vs $61.86 \pm 10.63 \mathrm{~cm})$.

Interestingly, a mixed-model ANOVA for repeated measures with "slightly uncomfortable distance" as a dependent 
Table 1. Demographic, neuropsychological, and personality characteristics of the subjects participating in Experiments 1 and 2

\begin{tabular}{|c|c|c|c|c|}
\hline & \multicolumn{2}{|l|}{ OXT group } & \multicolumn{2}{|l|}{ PLC group } \\
\hline & $\begin{array}{l}\text { Singles } \\
(n=14)\end{array}$ & $\begin{array}{l}\text { Relationship } \\
(n=14)\end{array}$ & $\begin{array}{l}\text { Singles } \\
(n=13)\end{array}$ & $\begin{array}{l}\text { Relationship } \\
(n=16)\end{array}$ \\
\hline Age (years) & $23.50(2.62)$ & $26.57(2.85)$ & $25.31(2.59)$ & $25.38(4.06)$ \\
\hline Years of education & $17.15(2.27)$ & $19.29(3.24)$ & $17.19(2.51)$ & $17.54(2.11)$ \\
\hline \multicolumn{5}{|l|}{ RAVLT } \\
\hline Trial 1-5 $5^{a}$ & $62.38(5.27)$ & $60.07(7.19)$ & $60.77(7.03)$ & $65.13(6.40)$ \\
\hline Trial 6: Retention ${ }^{b}$ & $14.31(1.18)$ & $13.64(1.55)$ & $13.77(3.06)$ & $13.87(1.41)$ \\
\hline Trial 7: Delayed recall & $14.77(0.44)$ & $13.86(1.17)$ & $13.69(3.30)$ & $13.93(1.44)$ \\
\hline LPS- $4^{d}$ & $32.46(3.13)$ & $32.36(3.27)$ & $31.00(4.49)$ & $31.80(3.93)$ \\
\hline MWT-A & $30.57(2.34)$ & $31.93(2.02)$ & $30.54(3.41)$ & $31.87(2.72)$ \\
\hline $\mathrm{d} 2^{f}$ & $205.14(40.13)$ & $195.55(28.41)$ & $183.69(38.38)$ & $202.13(29.25)$ \\
\hline TMT-A (seconds) & $26.62(6.78)$ & $26.50(9.66)$ & $21.89(4.43)$ & $25.03(6.29)$ \\
\hline TMT-B ${ }^{g}$ (seconds) & 59.14 (19.51) & 55.07 (16.81) & $64.78(19.25)$ & $56.52(21.64)$ \\
\hline Digit-span, forward ${ }^{h}$ & $8.79(2.19)$ & $9.21(1.85)$ & $9.46(1.85)$ & $9.06(2.38)$ \\
\hline Digit-span, backwards ${ }^{h}$ & $8.15(2.48)$ & $9.13(2.42)$ & $9.07(2.16)$ & $9.29(2.13)$ \\
\hline STAI State, pre $^{i}$ & $40.50(2.90)$ & $41.07(2.92)$ & $40.88(3.50)$ & $40.85(3.11)$ \\
\hline STAI State, post ${ }^{i}$ & $40.00(2.66)$ & $41.57(3.86)$ & $41.92(3.97)$ & $40.06(2.84)$ \\
\hline STAI Trait ${ }^{i}$ & $43.86(4.72)$ & $42.67(1.30)$ & $42.33(4.50)$ & $43.64(4.48)$ \\
\hline Positive affect, pre & $27.00(5.39)$ & $28.14(5.36)$ & $30.54(6.28)$ & $29.94(5.50)$ \\
\hline Positive affect, post $t^{j}$ & $26.64(6.13)$ & $27.79(6.41)$ & $29.54(5.32)$ & $28.69(5.49)$ \\
\hline Negative affect, pre $^{j}$ & $11.00(0.78)$ & $11.57(2.28)$ & $12.23(3.44)$ & $10.63(1.02)$ \\
\hline Negative affect, post ${ }^{j}$ & $10.86(1.29)$ & $11.29(1.86)$ & $10.63(1.26)$ & $11.15(1.63)$ \\
\hline SIAS ${ }^{k}$ & $14.29(7.53)$ & $15.43(8.24)$ & $13.00(6.95)$ & $11.94(5.27)$ \\
\hline SPS ${ }^{\prime}$ & $4.57(3.90)$ & $5.33(5.00)$ & $4.38(3.36)$ & $4.07(3.34)$ \\
\hline $\mathrm{BIS}^{m}$ & $2.07(0.42)$ & $2.18(0.41)$ & $2.16(0.52)$ & $2.37(0.56)$ \\
\hline $\mathrm{BAS}^{n}$ & $2.92(0.43)$ & $3.02(0.32)$ & $3.17(0.49)$ & $3.15(0.45)$ \\
\hline
\end{tabular}

There were neither main nor interaction effects of treatment and relationship status (all $p s>0.05)$ for any of the parameters listed in this table. Verbal declarative memory performance was assessed using a German adaption of the RAVLT (Rey Auditory Verbal Leaming Test) and included the following: ${ }^{a}$ learning performance across five trials (maximum possible score 75), ${ }^{b}$ susceptibility to interference (maximum possible score 15), and 'delayed recall (maximum possible score 15). Nonverbal reasoning IQ was assessed by the ${ }^{d}$ LS (Leistungsprüfsystem) subtest 4 (maximum possible score 40 ). Verbal IO based on lexical decisions was assessed by the ${ }^{e}$ MWT-A (Mehrfachwahl-Wortschatz-Intelligenz-Test Teil A; maximum possible score 37), visual attention and concentration was assesses using the $\mathrm{f} 2$ (Aufmerksamkeits- und Belastungstest d2), visual attention and task-switching was assessed using the ${ }^{9}$ TMT-A and TMT-B (Trail-making test $A$ and $B$ ), working memory performance was assessed using the ${ }^{h}$ digit-span forward and backward test (maximum possible score 14). Mood and anxiety were assessed before and after the experiment with the isTAI (State Trait Anxiety Inventory) and theipANAS (Positive and Negative Affective Schedule). Personality traits were measured with the ${ }^{k}$ SIAS (Social Interaction Anxiety Scale), the'SPS (Social Phobia Scale), the ${ }^{m}$ BIS (Behavioral Inhibition System), and the BAS (Behavioral Approach System). Given are mean values (SD)

variable showed a main effect of treatment $\left(F_{(1,53)}=4.93\right.$, $\left.p=0.03, \eta^{2}=0.09\right)$, but not of relationship status $\left(F_{(1,53)}=2.82\right.$, $\left.p=0.10, \eta^{2}=0.05\right)$ or interaction $\left(F_{(1,53)}=1.69, p=0.20, \eta^{2}=\right.$ $0.03)$. However, an exploratory analysis revealed that pairbonded men reported feeling uncomfortable at a greater distance from the female experimenter under OXT, but only if the latter was the one moving $\left(t_{(28)}=-3.43, p<0.01, \eta^{2}=0.30\right)$ and not if the subject was $\left(t_{(20.50)}=-1.97, p=0.06, \eta^{2}=0.13\right)$. For singles, there was no significant difference for either condition ( $p s \geq 0.44)$. Moreover, we detected a significant interaction between eye contact and person moving $\left(F_{(1,53)}=43.29, p<0.01\right.$, $\left.\eta^{2}=0.45\right)$. Returned eye contact compared with an averted gaze resulted in a larger distance if the experimenter was moving $(39.41 \pm 6.96 \mathrm{~cm}$ vs $37.95 \pm 6.96 \mathrm{~cm})$ but not if the subject was moving $(38.20 \pm 8.69 \mathrm{~cm}$ vs $39.78 \pm 8.41 \mathrm{~cm})$.

Further analysis showed that subjects with higher, but nonpathological social phobia scores maintained larger distances both in the ideal $(r=0.29, p=0.04)$ and in the slightly uncomfortable $(r=0.50, p<0.01$; Fig. 1 Aiv) condition, providing further validation that these measures reflect motivation to engage in a social encounter. All subjects rated the experimenter as highly likeable $(8.46 \pm 0.66)$ and trustworthy $(8.37 \pm 0.72)$, but after controlling for multiple comparisons (six ratings), we found no significant main or interaction effect of OXT and relationship status on reported feelings (all $p s \geq 0.08$ ). Thus, there was no
Table 2. Demographic, neuropsychological, and personality characteristics of the subjects participating in the independent control experiment

\begin{tabular}{|c|c|c|}
\hline & OXT $(n=15)$ & $\operatorname{PLC}(n=14)$ \\
\hline Age (years) & $26.40(3.29)$ & $25.43(3.24)$ \\
\hline Years of education & $18.43(2.53)$ & $17.64(2.27)$ \\
\hline \multicolumn{3}{|l|}{ RAVLT } \\
\hline Trial $1-5^{a}$ & $58.14(7.93)$ & $64.17(6.25)$ \\
\hline Trial 6: Retention ${ }^{b}$ & $12.29(2.20)$ & $13.67(1.50)$ \\
\hline Trial 7: Delayed recall ${ }^{c}$ & $12.71(1.94)$ & $13.92(1.51)$ \\
\hline LPS- $4^{d}$ & $30.93(3.27)$ & $31.31(3.79)$ \\
\hline MWT-A & $31.86(2.35)$ & $31.64(3.03)$ \\
\hline $\mathrm{d} 2^{f}$ & $210.91(25.38)$ & $221.55(24.24)$ \\
\hline TMT-A (seconds) & $24.50(7.38)$ & $23.44(6.87)$ \\
\hline TMT-B $^{g}$ (seconds) & $63.08(15.93)$ & $50.41(11.87)$ \\
\hline Digit-span, forward ${ }^{h}$ & $8.21(8.54)$ & $8.54(2.03)$ \\
\hline Digit-span, backwards ${ }^{h}$ & $7.86(2.28)$ & $7.93(2.14)$ \\
\hline STAI State, pre $^{i}$ & $43.07(2.87)$ & $41.29(2.46)$ \\
\hline STAI State, post ${ }^{i}$ & $41.93(3.41)$ & $39.57(4.86)$ \\
\hline STAI Trait ${ }^{i}$ & $43.07(4.46)$ & $41.14(3.67)$ \\
\hline Positive affect, pre & $30.80(4.07)$ & $28.36(5.03)$ \\
\hline Positive affect, post $t^{j}$ & $26.87(7.49)$ & $27.86(6.06)$ \\
\hline Negative affect, pre ${ }^{j}$ & $10.87(1.25)$ & $11.57(3.27)$ \\
\hline Negative affect, post ${ }^{j}$ & $11.00(1.60)$ & $10.71(0.83)$ \\
\hline SIAS ${ }^{k}$ & $11.57(3.60)$ & $12.25(5.60)$ \\
\hline SPS' & $3.50(3.21)$ & 4.29 (3.99) \\
\hline $\mathrm{BIS}^{m}$ & $2.01(0.37)$ & $2.21(0.66)$ \\
\hline BAS $^{n}$ & $3.27(0.18)$ & $2.93(0.47)$ \\
\hline
\end{tabular}

There were no significant between-group differences for any of the parameters listed in this table (all $p s>0.05$ ) The applied inventories and tests are referenced in Table 1. Given are mean values (SD).

Table 3. Ideal and slightly uncomfortable distances ( $\mathrm{cm}$ ) as measured for pair-bonded participants in the physical presence of a female (Experiment 1) and male (control experiment) experimenter

\begin{tabular}{|c|c|c|c|c|}
\hline & \multicolumn{2}{|c|}{ Female experimenter } & \multicolumn{2}{|c|}{ Male experimenter } \\
\hline & OXT $(n=14)$ & $\operatorname{PLC}(n=16)$ & OXT $(n=15)$ & $\operatorname{PLC}(n=14)$ \\
\hline \multicolumn{5}{|l|}{ Ideal distance } \\
\hline \multicolumn{5}{|c|}{ Experimenter moves } \\
\hline Far (EC) & $70.41(9.41)$ & $55.90(10.01)$ & $64.27(9.66)$ & $60.94(10.46)$ \\
\hline Far (NEC) & $67.96(9.93)$ & $54.11(8.66)$ & $63.28(9.80)$ & $58.95(10.00)$ \\
\hline Close (EC) & $68.40(7.31)$ & $57.56(10.02)$ & $72.16(7.55)$ & $64.54(10.86)$ \\
\hline Close (NEC) & $68.16(7.25)$ & $55.82(8.88)$ & $72.34(7.66)$ & $65.20(10.50)$ \\
\hline \multicolumn{5}{|c|}{ Participant moves } \\
\hline $\operatorname{Far}(\mathrm{EC})$ & $69.43(12.41)$ & $54.52(11.65)$ & $66.13(10.09)$ & $60.33(11.26)$ \\
\hline Far (NEC) & $68.86(12.06)$ & $55.56(11.71)$ & $64.96(9.59)$ & $60.45(11.75)$ \\
\hline Close (EC) & $78.41(15.13)$ & $61.27(11.03)$ & 71.07 (10.24) & $64.47(9.84)$ \\
\hline Close (NEC) & $79.31(14.11)$ & $60.56(10.84)$ & $71.00(10.79)$ & $65.02(10.95)$ \\
\hline \multicolumn{5}{|c|}{ Uncomfortable distance } \\
\hline \multicolumn{5}{|c|}{ Experimenter moves } \\
\hline Far (EC) & $44.40(5.44)$ & $37.47(4.38)$ & $38.82(4.18)$ & $37.75(5.26)$ \\
\hline Far (NEC) & $42.78(5.64)$ & $36.75(5.61)$ & $37.84(4.64)$ & $38.05(5.41)$ \\
\hline \multicolumn{5}{|c|}{ Participant moves } \\
\hline $\operatorname{Far}(\mathrm{EC})$ & $43.01(10.58)$ & $36.18(6.02)$ & $37.94(5.47)$ & $37.02(6.66)$ \\
\hline Far (NEC) & $43.74(10.52)$ & $37.92(6.59)$ & $39.26(5.61)$ & $37.85(6.53)$ \\
\hline
\end{tabular}

Overall, OXT significantly enlarged social distance (across all conditions) in the physical presence of a female experimenter $(p<0.01)$, but not of a male experimenter $(p=0.11)$. Given are mean values (SD). EC, Eye contact; NEC, no eye contact.

evidence that OXT was altering the perceived impression of the unfamiliar female by the pair-bonded men.

In the control experiment involving a male experimenter (cf. Table 3), the main and interaction effects observed with a female experimenter (Experiment 1) were absent (all $p s \geq 0.09$ ), indicating a specific role of the peptide in pair-bond maintenance. Participants of the control experiment rated the female experimenter (Experiment 1$)$ as attractive $(64.10 \pm 14.77)$, independent of treatment $\left(t_{(27)}=-0.81, p=0.42, \eta^{2}=0.02\right)$. 
In Experiment 2, we used the AA-task to test whether these OXT effects occurred in a more general context independent of the specific experimenter. A mixed-model ANOVA for repeated measurements with "valence" (positive or negative) and "content" (social or nonsocial) as within-subjects factors, "treatment" (OXT or PLC) and "relationship status" (pair-bonded or single) as between-subjects factors, and "reaction time" as a dependent variable yielded a main effect of valence $\left(F_{(1,53)}=6.97, p=0.01\right.$, $\left.\eta^{2}=0.12\right)$, an interaction between content, treatment, and relationship status $\left(F_{(1,53)}=17.00, p<0.01, \eta^{2}=0.24\right)$, and an interaction between valence, content, treatment, and relationship status $\left(F_{(1,53)}=4.91, p=0.03, \eta^{2}=0.09\right)$. Univariate ANOVAs with treatment and relationship status as independent variables and the reaction times for positive social, positive nonsocial, negative social, or negative nonsocial dependent variables showed only a significant interaction for positive social pictures between treatment and relationship status $\left(F_{(1,53)}=4.40, p=\right.$ $0.04, \eta^{2}=0.08$; Fig. $1 B$ ). Post hoc unpaired $t$ tests showed that the reaction time was significantly increased in pair-bonded men $\left(t_{(28)}=-2.17, p=0.04, \eta^{2}=0.14\right)$ but not in single ones $\left(t_{(25)}=\right.$ $\left.0.81, p=0.42, \eta^{2}=0.03\right)$. Similarly, Mann-Whitney tests revealed significantly more errors for pair-bonded men but not single ones following OXT treatment only in the positive social condition $(U=64, z=-2.09, p=0.04, r=-0.38$; Fig. $1 B)$, and not in positive nonsocial or negative social or nonsocial ones. There was no evidence for significant changes in emotional responsiveness to the pictures since the valence and arousal ratings were comparable across groups (all $p s \geq 0.08$ ). Overall, these results demonstrate that OXT treatment selectively impeded approach behavior toward attractive females in pair-bonded males either during a first social encounter (Experiment 1) or in the context of erotic IAPS pictures (Experiment 2), but without altering their judgments of how likeable or arousing the females were.

\section{Discussion}

These data substantially support the concept that the behavioral effects of OXT are moderated by contextual factors and personal characteristics such as relationship status (Bartz et al., 2011) and provide the first direct evidence for a behavior-modifying role of OXT in helping to maintain monogamous pair-bonds in humans. By selectively influencing men in a monogamous relationship to keep greater distance between themselves and attractive female strangers under physical presence as well as photographbased test conditions, OXT may act to promote fidelity. Importantly, since subjects did not report any significant changes in their impression or arousal evoked by female strangers, the observed effects of OXT cannot be attributed to a consciously altered attitude toward other women in general. The notion that the intrinsic reward value of other women remains constant is further supported by the absence of any interaction between OXT and gaze direction, since returned eye gaze from an attractive person is associated with a higher reward value than averted gaze when a social interaction is initiated (Kampe et al., 2001). While we originally considered that OXT might make single men approach more closely to women, we found no evidence for this, possibly suggesting that approach distances are already optimal for avoiding entering intimate space and therefore could not be further reduced.

In humans, monogamy may be less ecologically influenced than in other species and in many human societies, extra-pair copulations are sanctioned by social penalties of third parties (particularly kin) (Low, 2003). Thus, we avoided any measure- ments that could prime social norms and raise suspicion regarding our hypothesis. The AA-task was framed as a discrimination of valence and contained not only positive but also negative stimuli. Given that we found no evidence for a cognitive devaluation of other women, we consider it unlikely that our results are due to a stronger norm compliance of pair-bonded men.

The specificity of the OXT effect in influencing the social distance that males keep from unfamiliar females is evidenced by our finding that it has no significant effect on the distance kept from unfamiliar males. Given the absence of PS differences between single and pair-bonded men in the PLC group, it is clear that for these potential fidelity-enhancing effects of OXT to be revealed, female partners would need to evoke its endogenous release immediately before contexts in which the men might encounter other women. While, compared with singles, basal concentrations of OXT in blood are increased in couples during the early stages of romantic love and stay significantly elevated in couples remaining together 6 months later (Schneiderman et al., 2012), our results suggest that further augmentation of endogenous OXT release is necessary to produce fidelity-enhancing effects. Mechanistically, this may be related to additional OXT either compensating a relative deficit (optimization hypothesis) or by collapsing already optimal levels (decompensation hypothesis). While the most obvious physiological stimulus for promoting endogenous OXT release in men would be having sex with their mate (Krüger et al., 2003), the simple close presence and touch of their partner at any given moment in time might also suffice (Holt-Lunstad et al., 2008). Thus, OXT effects in promoting monogamy in males may normally depend upon the presence of a close positive relationship in the bond with their female partners and close physical proximity between the couple.

There also may be conditions where OXT increases PS regardless of relationship status. If the female experimenter started distally and kept eye contact while moving forward, it seems that singles in the OXT group also exhibited slightly larger distances (Fig. 1 Aii). However, given the absence of overall interaction effects between treatment and starting position or eye contact, and the three times larger variance of PS in OXT-treated singles $(\mathrm{SD}=29.03)$ compared with pair-bonded men $(\mathrm{SD}=9.41)$, this apparent difference is likely caused by outliers.

Future studies are needed to elucidate the exact neurochemical cascade underlying the observed OXT effect on approach behavior since OXT-induced analgesia in mice, for example, is mediated by vasopressin-1A receptors (Schorscher-Petcu et al., 2010). Similarly, an analysis of which of the various aspects of a pair-bond potentially interact with OXT to promote fidelity in monogamous males may constitute a promising approach to explore the fascinating, albeit tremendously complex, neurobiology of human pair-bonding.

\section{References}

Argyle M, Dean J (1965) Eye-contact, distance and affiliation. Sociometry 28:289-304.

Bartz JA, Zaki J, Bolger N, Ochsner KN (2011) Social effects of oxytocin in humans: context and person matter. Trends Cogn Sci 15:301-309.

Declerck CH, Boone C, Kiyonari T (2010) Oxytocin and cooperation under conditions of uncertainty: the modulating role of incentives and social information. Horm Behav 57:368-374.

De Dreu CK (2012) Oxytocin modulates cooperation within and competition between groups: an integrative review and research agenda. Horm Behav 61:419-428.

Ditzen B, Schaer M, Gabriel B, Bodenmann G, Ehlert U, Heinrichs M (2009) Intranasal oxytocin increases positive communication and reduces cortisol levels during couple conflict. Biol Psychiatry 65:728-731. 
Donaldson ZR, Young LJ (2008) Oxytocin, vasopressin, and the neurogenetics of sociality. Science 322:900-904.

Feldman R (2012) Oxytocin and social affiliation in humans. Horm Behav 61:380-391.

Gamer M, Zurowski B, Büchel C (2010) Different amygdala subregions mediate valence-related and attentional effects of oxytocin in humans. Proc Natl Acad Sci U S A 107:9400-9405.

Guastella AJ, Mitchell PB, Dadds MR (2008) Oxytocin increases gaze to the eye region of human faces. Biol Psychiatry 63:3-5.

Hall ET (1966) The hidden dimension. Garden City, NY: Doubleday.

Hayduk LA (1983) Personal space: where we now stand. Psychologic Bull 94:293-335.

Holt-Lunstad J, Birmingham WA, Light KC (2008) Influence of a "warm touch" support enhancement intervention among married couples on ambulatory blood pressure, oxytocin, alpha amylase, and cortisol. Psychosom Med 70:976-985.

Hurlemann R, Patin A, Onur OA, Cohen MX, Baumgartner T, Metzler S, Dziobek I, Gallinat J, Wagner M, Maier W, Kendrick KM (2010) Oxytocin enhances amygdala-dependent, socially reinforced learning and emotional empathy in humans. J Neurosci 30:4999-5007.

Insel TR (2010) The challenge of translation in social neuroscience: a review of oxytocin, vasopressin, and affiliative behavior. Neuron 65:768-779.

Kampe KK, Frith CD, Dolan RJ, Frith U (2001) Reward value of attractiveness and gaze. Nature 413:589.

Kemp AH, Guastella AJ (2011) The role of oxytocin in human affect: a novel hypothesis. Curr Dir Psychol Sci 20:222-231.

Kennedy DP, Gläscher J, Tyszka JM, Adolphs R (2009) Personal space regulation by the human amygdala. Nat Neurosci 12:1226-1227.

Krüger TH, Haake P, Chereath D, Knapp W, Janssen OE, Exton MS, Sched- lowski M, Hartmann U (2003) Specificity of the neuroendocrine response to orgasm during sexual arousal in men. J Endocrinol 177:57-64.

Lang PJ, Bradley MM, Cuthbert BN (2005) International affective picture system (IAPS): affective ratings of pictures and instruction manual. Technical Report A-6. Gainesville, FL: University of Florida.

Low BS (2003) Ecological and social complexities in human monogamy. In: Mating strategies and partnerships in birds, humans, and other mammals (Reichard UH, Boesch C, eds). Cambridge, UK: Cambridge UP.

Mattick RP, Clarke JC (1998) Development and validation of measures of social phobia scrutiny fear and social interaction anxiety. Behav Res Ther 36:455-470.

McCall C, Singer T (2012) The animal and human neuroendocrinology of social cognition, motivation and behavior. Nat Neurosci 15:681-688.

Meyer-Lindenberg A, Domes G, Kirsch P, Heinrichs M (2011) Oxytocin and vasopressin in the human brain: social neuropeptides for translational medicine. Nat Rev Neurosci 12:524-538.

Schneiderman I, Zagoory-Sharon O, Leckman JF, Feldman R (2012) Oxytocin during the initial stages of romantic attachment: relations to couples' interactive reciprocity. Psychoneuroendocrinology 37:1277-1285.

Schorscher-Petcu A, Sotocinal S, Ciura S, Dupré A, Ritchie J, Sorge RE, Crawley JN, Hu SB, Nishimori K, Young LJ, Tribollet E, Quirion R, Mogil JS (2010) Oxytocin-induced analgesia and scratching are mediated by the vasopressin-1A receptor in the mouse. J Neurosci 30:8274-8284.

Striepens N, Kendrick KM, Maier W, Hurlemann R (2011) Prosocial effects of oxytocin and clinical evidence for its therapeutic potential. Front Neuroendocrinol 32:426-450.

Sunnafrank M, Ramirez AJ (2004) At first sight: persistent relational effects of get-acquainted conversations. J Soc Pers Relat 21:361-379. 